\title{
Velocity Field Modelling for Pollutant Plume Using 3-D Adaptive Finite Element Method ${ }^{\star}$
}

\author{
Gustavo Montero, Rafael Montenegro, José María Escobar, \\ Eduardo Rodríguez, and José María González-Yuste
}

University Institute of Intelligent Systems and Numerical Applications in Engineering, Univ. of Las Palmas de Gran Canaria, Edif. Central Parque Científico-Tecnológico, Campus Universitario de Tafira, 35017 Las Palmas de Gran Canaria, Spain

\{rafa,gustavo\}@dma.ulpgc.es, jescobar@dsc.ulpgc.es, barrera@dma.ulpgc.es, jmgonzalezy@pas.ulpgc.es

\begin{abstract}
Air pollution models usually start from the computation of the velocity field of a fluid. In this paper, we present a model for computing that field based on the contribution of the observed wind flow and the vertical buoyancy or momentum plume rise defined by a Gaussian plume model. This initial velocity field is adjusted to verify incompressibility and impermeability conditions by using a mass consistent model. In this environmental modelling that occur in a three-dimensional domain defined over complex terrain, a mesh generator capable of adapting itself to the topographical data and to the numerical solution is essential. Here, the unstructured tetrahedral meshes are generated by combining the use of a refinement/derefinement algorithm for two-dimensional domains and a tetrahedral mesh generator based on Delaunay triangulation. Occasionally in this process, low quality or even inverted elements may appear. A simultaneous untangling and smoothing procedure allows to optimise the resulting meshes. Once we have constructed the adapted mesh in accordance with the geometrical characteristics of our domain, we use an adaptive local refinement in the plume trajectory. Finally, this model is applied in a test problem.
\end{abstract}

\section{Introduction}

In [1] the authors propose a mesh generator for environmental problems which is applied in this model. The studied domain is limited at the bottom by the terrain and at the top by a horizontal plane. The lateral walls are formed by four vertical planes. Specifically, we propose the construction of a regular triangulation of this upper boundary. Now, the refinement/derefinement algorithm [2] is applied over this regular mesh to define an adaptive node distribution of the layer corresponding to the terrain surface and the functions which represent the chimneys. Once the node distribution is defined on the terrain and the upper boundary, we begin to distribute the nodes located between both layers by using a vertical spacing function. Next, a three-dimensional mesh generator based on

\footnotetext{
* Supported by MCYT and FEDER. Grant contract: REN2001-0925-C03-02/CLI
} 
Delaunay triangulation [3] is applied. In a first stage, our 3-D mesh generator constructs meshes with poor quality and, in special cases, for example when node movement is required, inverted elements may appear. In [4] we propose a procedure for mesh untangling and smoothing in the same stage. In addition, along the plume trajectory, we apply a local refinement algorithm [5] based on the 8subtetrahedron subdivision. This tetrahedral mesh is used in a mass consistent model for wind field adjustment [6]. To obtain the observed wind, horizontal interpolation of the station measures is carried out. Then, a log-linear wind profile is built in the surface layer taking into account the horizontal interpolation, the effect of roughness and the air stability. Above the surface layer, a linear interpolation is carried out using the geostrophic wind.

\section{Vertical Velocity Correction along Plume Trajectory}

The main idea is to add a vertical velocity along the trajectory of a pollutant plume arising from a chimney to the interpolated wind field, which usually only consider the horizontal components of wind velocities. Gaussian plume models allow to approximate the effective height of a plume $z_{H}$ and the horizontal distance $d_{f}$ from the stack to the point where the plume height reaches $z_{H}$, depending on the emission characteristics, the wind and the atmospheric stability. Gases rise from the stack if their density is lower than the air density (buoyancy rise) or due to emission velocity (momentum rise). In order to compute the effective height of the plume, we use Briggs' equations (see e.g. [7]). So, using the computed values of $z_{H}$ and $d_{f}$ for buoyancy rise, except for stable conditions and calm wind, we propose to adjust the vertical component of the velocity along the trajectory of the plume by a linearly unaccelerated motion. In addition, the horizontal motion from the source to the distance $d_{f}$ is considered uniformly accelerated. Thus, the time $t_{f}$ corresponding to the distance $d_{f}$ is,

$$
t_{f}=\frac{1}{a_{d}}\left(-\left|\boldsymbol{v}_{0}\left(x_{c}, y_{c}, z_{c}\right)\right|+\sqrt{\left|\boldsymbol{v}_{0}\left(x_{c}, y_{c}, z_{c}\right)\right|^{2}+2 a_{d} d_{f}}\right)
$$

where $a_{d}$ is the module of horizontal acceleration $\left(a_{d x}, a_{d y}\right)$ in the direction of wind velocity $\boldsymbol{v}_{0}$ at the centre of the emission surface $\left(x_{c}, y_{c}, z_{c}\right)$. The trajectory of the plume, the vertical velocity $w_{0}$ and the vertical acceleration $a_{0}$, are then given by the following functions of the parameter $t$,

$$
\begin{aligned}
x(t) & =x_{c}+u_{0}\left(x_{c}, y_{c}, z_{c}\right) t+\frac{1}{2} a_{d x} t^{2} \\
y(t) & =y_{c}+v_{0}\left(x_{c}, y_{c}, z_{c}\right) t+\frac{1}{2} a_{d y} t^{2} \\
z(t) & =z_{c}^{\prime}+w_{c} t+K_{1} t^{2}+K_{2} t^{3} \\
w_{0}(t) & =w_{c}+2 K_{1} t+3 K_{2} t^{2} \\
a_{0}(t) & =2 K_{1}+6 K_{2} t
\end{aligned}
$$

where $K_{1}=\frac{-2 w_{c} t_{f}+3\left(z_{H}-z_{c}^{\prime}\right)}{t_{f}^{2}}, K_{2}=\frac{w_{c} t_{f}-2\left(z_{H}-z_{c}^{\prime}\right)}{t_{f}^{3}}$, with $w_{c}$, the emission velocity of gases and $z_{c}^{\prime}$, the effective height of the stack. The vertical component of 
velocity $\boldsymbol{v}_{0}$ is modified in any point of the domain $\Omega$ located inside a cylinder generated by the circular emission surface of the gases (of diameter $D_{c}$ ) which is moving parallel to the horizontal plane, along the parametric curve given by equations (2), (3) and (4) between $t=0$ and $t=t_{f}$. Thus, constant vertical velocities are generated in the cylinder for each horizontal disk.

On the other hand, for momentum rise or buoyancy rise with stable condition and calm wind, the horizontal motion of the plume until reaching the effective height is very small. Thus the trajectory of the gases is nearly vertical. In this case, we consider a uniformly unaccelerated motion. Now $t_{f}=\frac{2}{w_{c}}\left(z_{H}-z_{c}^{\prime}\right)$ and the acceleration $a_{0}=\frac{-w_{c}}{t_{f}}$. Thus, the vertical velocity at a point of height $z$ is $w_{0}(z)=w_{c} \sqrt{1-\frac{2\left(z-z_{c}^{\prime}\right)}{w_{c} t_{f}}}$. Here, the vertical component of the velocity is modified inside a standard cylinder of which base is the emission surface of gases in the stack and its height is $z_{H}-z_{c}^{\prime}$.

\section{$3 \quad$ Numerical Experiments}

For air pollution modelling of a test power plant located in the region of La Palma Island, we add the chimney geometry to the topographical data and apply the 3-D mesh generator. Consider a chimney with a height of $200 \mathrm{~m}$ over the terrain and diameter of $20 \mathrm{~m}$ at its top and $40 \mathrm{~m}$ at its bottom. Since, the mesh must be able to detect the details of the chimney, a size of elements about $2 \times 2 \mathrm{~m}$ in the chimney, starting from the uniform 2-D mesh $\tau_{1}$ of the rectangular area with a size of elements about $2 \times 2 \mathrm{~km}$, means to make ten global refinement steps using Rivara 4-T algorithm [8]. However, we only need five global refinement steps over $\tau_{1}$ and, after, five local refinement steps of elements inside the chimney.

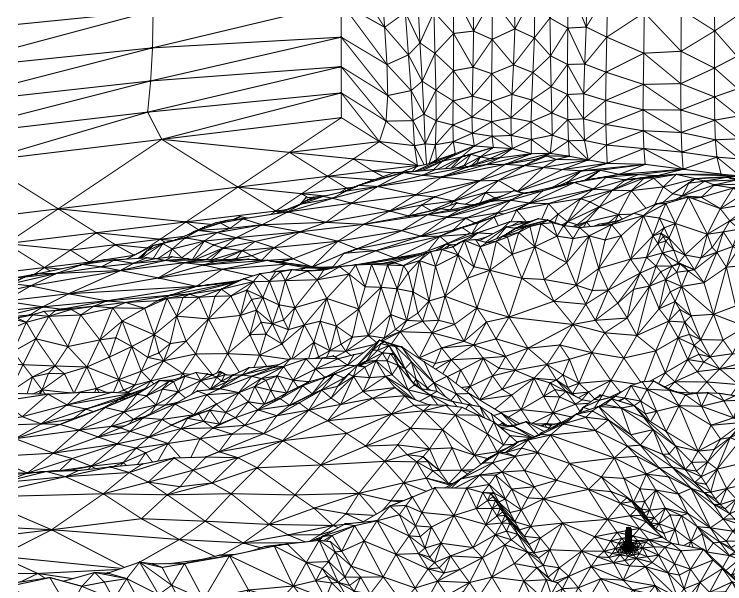

Fig. 1. Detail of a 3-D mesh with a chimney near the right bottom corner 


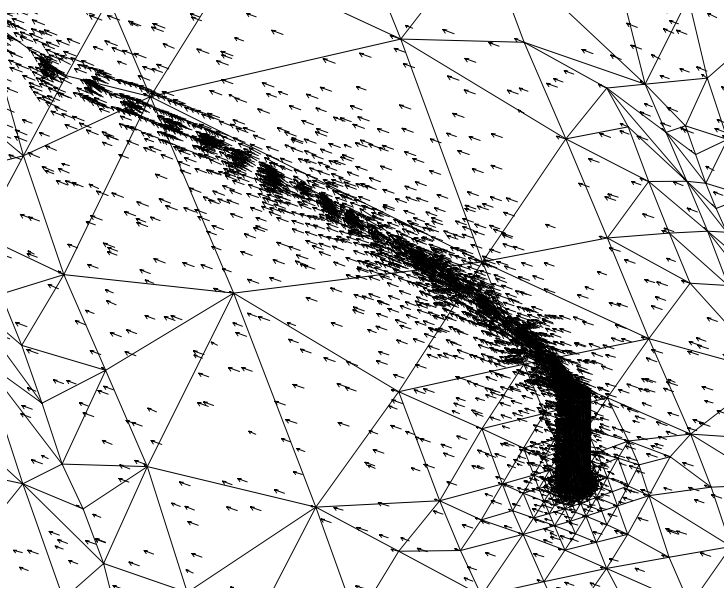

Fig. 2. Detail of the simulated velocity field in the surroundings of the chimney

Here we applied the derefinement algorithm with a parameter $\varepsilon=40 \mathrm{~m}$ considering that those nodes located inside the chimney could not be eliminated. Finally, we have applied six local refinement steps in the plume trajectory to previous resulting 3-D mesh in order to obtain a new mesh with 31555 nodes and 170784 tetrahedra. Figs. 1 1 and 2 show a detail of the mesh and the adjusted velocity field where the effect of chimney emission is included, respectively.

\section{References}

1. Montenegro, R., Montero, G., Escobar, J.M., Rodríguez, E., González-Yuste, J.M.: Tetrahedral Mesh Generation for Environmental Problems over Complex Terrains. Lect. Notes Comp. Sci., 2329 (2002) 335-344

2. Ferragut, L., Montenegro, R., Plaza, A.: Efficient Refinement/Derefinement Algorithm of Nested Meshes to Solve Evolution Problems. Comm. Numer. Meth. Engng. 10 (1994) 403-412

3. Escobar, J.M., Montenegro, R.: Several Aspects of Three-Dimensional Delaunay Triangulation. Adv. Engng. Soft. 27(1/2) (1996) 27-39

4. Escobar, J.M., Rodríguez, E., Montenegro, R., Montero, G., González-Yuste, J.M.: Simultaneous Untangling and Smoothing of Tetrahedral Meshes. Comp. Meth. Appl. Mech. Engng. 192 (2003) 2775-2787

5. González-Yuste, J.M., Montenegro, R., Escobar, J.M., Montero, G., Rodríguez, E.: An Object Oriented Method for Tetrahedral Mesh Refinement. Adv. Engng. Soft., in press.

6. Montero, G., Montenegro, R., Escobar, J.M.: A 3-D Model for Wind Field Adjustment. J. Wind Engng. \& Ind. Aer. 74-76 (1998) 249-261

7. Boubel, R.W., Fox, D.L., Turner, D.B., Stern, A.C.: Fundamentals of Air Pollution. Academic Press, San Diego, (1994)

8. Rivara, M.C.: A Grid Generator Based on 4-Triangles Conforming. MeshRefinement Algorithms. Int. J. Num. Meth. Engng. 24 (1987) 1343-1354 